\section{Regards sur l'économie allemande}

Bulletin économique du CIRAC

$80 \mid 2007$

Varia

\title{
Présidence allemande de l'UE : convaincre et rassembler
}

Isabelle Bourgeois

\section{OpenEdition}

Édition électronique

URL : http://journals.openedition.org/rea/680

DOI : $10.4000 /$ rea.680

ISBN : 978-2-8218-0856-0

ISSN : 1965-0787

Éditeur

CIRAC

Édition imprimée

Date de publication : 1 mars 2007

Pagination : 5-10

ISSN : 1156-8992

Référence électronique

Isabelle Bourgeois, «Présidence allemande de l'UE : convaincre et rassembler », Regards sur l'économie allemande [En ligne], 80 I mars 2007, document 1, mis en ligne le 01 mars 2009, consulté le 30 avril 2019. URL : http://journals.openedition.org/rea/680; DOI : 10.4000/rea.680 


\title{
Présidence allemande de 1'UE : convaincre et rassembler
}

\author{
Isabelle Bourgeois
}

Coïncidence de l'agenda mondial, l'Allemagne, première économie européenne et $3^{e}$ mondiale, assume en ce premier semestre une double présidence : celle de l'UE et celle du G8. Les missions qui lui incombent dans ce cadre sont lourdes : relance du cycle de Doha, gestion des crises internationales dans une approche de coopération entre l'UE, I'OTAN et les Nations Unies, approfondissement d'une UE comprenant désormais 27 membres. Les attentes vis-à-vis de l'Allemagne sont d'autant plus grandes que l'urgence de l'organisation d'un marché mondial où se profilent de nouveaux acteurs intervient à un moment où l'Europe traverse la crise la plus profonde de son histoire. Non seulement le processus de Lisbonne est enlisé, ce qui bride la dynamique de croissance et la compétitivité de l'Union dans un environnement concurrentiel aujourd'hui globalisé. Mais l'Europe est surtout paralysée en tant qu'entité politique après une vague d'élargissement beaucoup trop rapide en comparaison de structures institutionnelles, révisées pour la dernière fois par le Traité de Nice de 2000, mais dès l'époque à peine performantes pour une UE à 15 membres seulement. Le "non" français et néerlandais au Traité constitutionnel qui devait notamment faire de l'UE une personnalité juridique a repoussé sine die le processus d'approfondissement, indispensable pourtant pour sceller l'unité de l'Europe.

Voilà donc le double défi majeur que doit relever l'Allemagne au cours de sa présidence de l'UE : impulser une nouvelle vie à la Stratégie de Lisbonne et au projet de Traité constitutionnel. Elle ne prend pas ses responsabilités à la légère ; l'histoire lui a appris combien elle est redevable à l'intégration européenne. Mais elle est consciente que cette responsabilité ne peut être exercée que collectivement: en interne, grâce à la coopération entre Bund, Länder, Bundestag, partis politiques et partenaires sociaux; au sein de l'UE, grâce à la contribution active de tous les autres Etats membres. C'est cela que traduit le choix de la devise "ensemble pour l'Europe " : étant donné la complexité des dossiers et des intérêts en jeu, l'Allemagne ne peut concevoir son leadership que comme une mission d'impulsion et, plus encore, de médiation.

\section{Une présidence différente des autres}

La présidence allemande tombe à un moment historique. Le 25 mars, l'Europe fêtera les 50 ans de son acte fondateur : les Traités de Rome (CEE et EURATOM), signés par les 6 Etats membres d'alors, qui créent un espace économique intégré reposant sur le principe de la libre circulation des biens, des services, des capitaux et des personnes. Ces textes, plusieurs fois amendés depuis, figurent dans le chapitre III du Traité constitutionnel en cours de ratification. Le 7 février, l'Europe a aussi fêté les 15 ans du Traité instituant l'Union européenne (UE), signé à Maastricht par les 12 Etats membres qu'elle comptait en 1992. Ce Traité transforme l'ancienne Communauté économique européenne (CEE) en une Communauté européenne (CE) caractérisée par l'introduction de la citoyenneté européenne et le renforcement des pouvoirs du Parlement européen. A ce premier pilier (CE/CECA/EURATOM) s'en ajoutent deux nouveaux: une politique étrangère et de sécurité commune (PESC) et une coopération en matière de justice et d'affaires intérieures. Ces trois piliers sont 
Une responsabilité particulière, mais pas de miracles à attendre

Priorité : se donner les moyens d'agir grâce au « Trio présidentiel »

Créer les conditions pour faire aboutir des processus complexes réunis au sein d'une sorte de holding : l'Union européenne (UE). Par ailleurs, le Traité de Maastricht instaure l'Unité économique et monétaire (UEM) qui a pris effet le $1^{\mathrm{er}}$ janvier 1999. Et il lance le projet d'un Traité constitutionnel chargé de se substituer aux grands textes fondateurs de l'Europe et d'en poursuivre l'approfondissement ; celui-ci, signé le 29 octobre 2004, a été ratifié jusqu'ici par 18 Etats membres sur les 27 que compte l'UE depuis le $1^{\text {er }}$ janvier 2007.

Coïncidence du calendrier des présidences tournantes du Conseil, l'honneur de célébrer ces étapes revient à un pays dont l'intégration dans l'Europe a favorisé I'Unité, celle-ci entraînant à sa suite la réunification de l'Europe après la chute du Rideau de Fer, concrétisée par l'intégration de 12 Etats membres est-européens. Reflet de ces évolutions, l'honneur revient à une chancelière elle-même originaire de l'ex-RDA. Si la conscience historique de l'Allemagne fonde sa responsabilité particulière pour l'Europe, la puissance des symboles et les attentes qu'elle développe chez ses partenaires n'en obèrent pas pour autant une approche pragmatique et, plus encore, réaliste, de la mission qui lui incombe alors même que l'Europe traverse une grave phase de doute. "Nous ne pourrons pas faire de miracles », insistait dès la fin 2006 Frank-Walter Steinmeier (SPD), ministre fédéral des Affaires étrangères (Süddeutsche Zeitung, 21-12-06).

Sous la direction d'une Angela Merkel européenne par conviction à la différence d'un Gerhard Schröder européen par nécessité, la première démarche de l'Allemagne a été de se donner les moyens d'assumer au mieux et dans l'intérêt collectif la difficile mission qui lui incombe. Pour pallier les insuffisances du Traité de Nice, elle a innové au plan institutionnel en créant un «Trio présidentiel ». Elle travaille ainsi depuis le $1^{\text {er }}$ janvier en étroite coopération avec les deux Etats qui lui succèderont à la tête du Conseil européen : le Portugal (second semestre 2007) et la Slovénie (premier semestre 2008). Cette nouvelle approche garantit la continuité de la politique menée, puisque celle-ci s'étend ainsi sur 18 mois au lieu des 6 réservés à un seul Etat. Elle présente en outre l'avantage d'associer deux 'petits' pays dont un nouvel entrant, ce qui permet aussi de mieux concilier les intérêts des 'grands' comme des 'petits' après la phase d'exacerbation des particularismes postérieure au Traité de Nice. Ainsi, le «Trio présidentiel » semble le seul moyen adapté pour faire face au nouveau programme du Conseil, échelonné sur 18 mois à compter de janvier 2007. II donne aussi le temps nécessaire pour désormais envisager concrètement l'avenir de l'Europe après la phase de paralysie déclenchée en 2005 par le refus du Traité constitutionnel par la France et les Pays Bas.

L'agenda, chargé, est organisé en deux temps par le rythme des sommets. D'ici le sommet de printemps (Bruxelles, 8 et 9 mars), et même le sommet informel qui se tiendra à Berlin à l'occasion du $50^{\circ}$ anniversaire des Traités de Rome (24 et 25 mars), l'agenda est dominé par les dossiers économiques - principalement la relance du processus de Lisbonne et l'élaboration d'une politique énergétique communautaire. Ensuite, il sera dominé par la question de l'avenir du Traité constitutionnel: lors du sommet des 21 et 22 juin à Bruxelles, l'Allemagne compte présenter une feuille de route pour la relance du processus de ratification, à charge pour le Portugal, la Slovénie puis la France (second semestre 2008) de faire aboutir les négociations de manière à ce que la réforme institutionnelle de l'UE soit achevée avant les élections au Parlement européen en 2009. Ce programme, inscrit dans la continuité de la présidence finlandaise, mais également 'imposé' par les conclusions de la présidence (Conseil européen de Bruxelles les 14 et 15 décembre 2006), de même que par le programme de travail de la Commission et divers programmes pluri-annuels, laisse peu de place aux initiatives nationales (programme 'libre') que peuvent prendre les Etats assumant la présidence du Conseil. II en laisse d'autant moins à l'Allemagne que sa présidence intervient à un moment où il s'agit moins de définir de nouveaux projets ou objectifs que de créer les conditions pour faire aboutir des processus hautement complexes. Et surtout, de redonner du sens à l'Europe. 
La difficulté consiste, pour l'Allemagne, à parler d'une seule voix. Elle ne provient pas de la structure de grande coalition que présente le gouvernement fédéral : à ce niveau politique, CDU/CSU et SPD partagent et respectent le Contrat de coalition. Elle réside en premier lieu dans la répartition institutionnelle des rôles entre A. Merkel et ses ministres. Si le chancelier "fixe les grandes orientations de la politique et en assume la responsabilité ", chaque ministre " dirige son département de façon autonome et sous sa propre responsabilité " (art. 65 de la Loi fondamentale). Au plan national, cela exige une intense coordination : horizontale au niveau interministériel, mais aussi dans le partage des rôles entre la chancelière et son ministre des Affaires étrangères, qui se traduit par l'étroite coopération de leurs états-majors institués pour mener eux aussi la coordination des dossiers européens. Au niveau vertical, les acteurs doivent rechercher l'équilibre des intérêts : si les ministres sont soumis au respect des orientations de la politique européenne allemande - fixées conjointement au sein du conseil des ministres -, la chancelière respecte en contrepartie leur latitude de décision en n'interférant pas dans leur action. Cette architecture trouve son reflet à l'échelon de l'UE où chaque ministre préside, au nom du pays assumant la présidence, les Conseils des ministres 'techniques' : Ecofin, Compétitivité ou encore Transports, Télécommunications et Energie. Dans cette fonction, il est habilité à engager son gouvernement. La coordination s'impose donc à cet échelon aussi. Une autre source de complexité réside dans le rôle prééminent du Bundestag dans le partage des pouvoirs outre-Rhin, les parlementaires allemands s'emparant volontiers des dossiers législatifs communautaires. Et au Parlement européen (PE), les députés allemands n'hésitent pas à défendre des positions indépendantes de celles du gouvernement fédéral.

Autre source de complexité : la structure fédérale de l'Allemagne. Les Länder, largement souverains sur leur territoire en matière de politique structurelle, mènent eux aussi leur propre politique européenne (chacun de leurs gouvernements comprend un ministre des Affaires européennes) - une autonomie trouvant son reflet à l'échelon de l'UE depuis la création en 1994 du Comité des Régions, un organe qui doit être consulté en amont de toute décision réglementaire concernant la politique économique et sociale régionale. A cela s'ajoute l'autonomie constitutionnelle des partenaires sociaux. Si ceux-ci, représentés au sein du Comité économique et social européen (CESE), ne disposent à l'échelon de l'UE que du pouvoir consultatif dévolu à cette institution, dans le jeu institutionnel allemand en revanche, ils sont seuls responsables de la macrorégulation sociale ; le gouvernement est constitutionnellement incompétent en la matière. La politique intérieure que mènent les partenaires sociaux allemands implique donc qu'ils se préoccupent des enjeux européens. Les décisions relatives au cadre des activités adoptées dans les diverses instances communautaires les concernent en effet au premier chef puisqu'elles déterminent l'évolution du cadre national des activités et donc leurs choix contractuels.

Pour concilier l'intérêt général communautaire et ses intérêts nationaux particuliers, l'Allemagne recourt à sa traditionnelle méthode de négociation sur la base d'un processus de co-décision. Fait nouveau, elle intègre dans ce processus collectif désormais explicitement la société civile, sans l'implication de laquelle aucune politique européenne n'est possible. Mais la nécessité de concilier les intérêts les plus divers n'est un handicap qu'à première vue. Car la structure même des processus décisionnels allemands fonde une culture de quête permanente du compromis, par nature adaptée à une architecture européenne au moins tout aussi complexe. C'est sur cette méthode et cette 'intelligence de la concertation' que repose une grande partie des attentes envers l'Allemagne.

\section{Refonder l'économie sociale de marché européenne}

La première priorité thématique de la présidence est la relance de la Stratégie de Lisbonne, largement restée lettre morte malgré une révision opérée en 2005.
Des processus nationaux complexes : autonomie des ministres fédéraux, ...

... souveraineté des Länder et des partenaires sociaux

Une culture de la négociation en adéquation avec les processus décisionnels européens

Relance de la Stratégie de Lisbonne 
L'Allemagne donne l'exemple...

... en assumant sa responsabilité économique en Europe

Priorité au parachèvemen du marché intérieur

Plaidoyer pour la simplification de l'acquis communautaire
Son objectif, défini lors du Conseil européen de Lisbonne en mars 2000, est de refonder le modèle économique et social européen, hérité de l'ère industrielle, pour l'adapter aux impératifs de l'économie du savoir et de la connaissance et garantir ainsi la compétitivité de l'Europe dans un contexte mondial en profonde mutation. Cette Stratégie, établie sur la base d'une logique contractuelle, suit une approche globale de modernisation du cadre des activités dans le but de générer de l'emploi et de préserver l'avenir de la protection sociale, ce que résume de manière simplifiée la devise officielle: "de la croissance, des emplois ». Sur la base des grands objectifs conjoints, visant tous à rétablir cet équilibre entre prospérité et solidarité au cœur du principe de «l'économie sociale de marché » qui fonde la construction européenne depuis les Traités de Rome, chaque Etat membre définit sa propre politique de modernisation en fonction de la situation nationale. Sa liberté de choix des moyens s'applique aussi à des objectifs transversaux comme une politique d'innovation devant se traduire entre autres par une part de la R\&D dans le PIB de $3 \%$ en 2010.

Or l'Allemagne est un des rares Etats membres à avoir commencé réellement à mettre en œuvre la Stratégie de Lisbonne et à s'en réclamer ouvertement pour expliquer les réformes structurelles nécessaires. L'Agenda 2010 présenté en mars 2003 par le gouvernement Schröder et dans la continuité duquel s'inscrivent les réformes du gouvernement Merkel commence à porter ses fruits, comme en atteste le retour de la dynamique de croissance outre-Rhin et l'embellie notable sur le marché de l'emploi. Le fait que la première économie européenne respecte à nouveau les termes du Pacte de stabilité et de croissance (même si les efforts de consolidation budgétaire sont encore insuffisants), contribue lui aussi à asseoir la légitimité de sa politique européenne dans ce domaine. "L'Europe sera un succès si chacun d'entre nous fait ses devoirs ", expliquait la chancelière dans son discours télévisé du Nouvel an, avant d'ajouter : " En 2007, il nous faudra redoubler d'efforts - pour faire progresser l'Europe, mais d'abord pour consolider notre propre croissance économique ".

Ces propos expriment la responsabilité européenne assumée de l'Allemagne, qui repose sur un double constat : la compétitivité et la prospérité de l'Europe dépendent largement du dynamisme de sa principale économie ; et c'est parce que les économies des Etats membres ont atteint aujourd'hui un haut degré d'interdépendance que l'Allemagne renoue avec la compétitivité. II est vrai que l'Allemagne a une approche naturelle de cette solidarité que génère une concurrence ordonnée : elle n'est que la transposition, à l'échelle de l'Europe, du mode de fonctionnement systémique interne à un pays dont la performance économique globale résulte de l'émulation de ses 'Etats membres' que sont les Länder. Il suffit que certains d'entre eux donnent des signes de faiblesse pour que la croissance de la RFA s'en trouve immédiatement tassée. A l'inverse, la compétitivité d'une Bavière ou d'une Saxe profite à la collectivité qu'est la RFA.

Très pragmatiquement, l'Allemagne peut donc mettre à profit sa propre expérience du processus de Lisbonne pour ré-impulser la dynamique de changement nécessaire au sein de l'UE. Lors du Sommet de mars, la Stratégie de Lisbonne sera évaluée, voire adaptée en fonction du bilan. Un des points portera sur le réalisme de l'objectif des $3 \%$ de R\&D dans le PIB ; l'Allemagne est le pays qui s'en rapproche le plus, mais ne parviendra pas elle non plus à le tenir d'ici 2010. Un autre point concerne le parachèvement du marché intérieur sous l'angle de la liberté de circulation des biens et des services. Pour une économie aussi dépendante du marché communautaire que l'Allemagne, il s'agit d'une priorité absolue. Et si ce dossier est 'imposé' par le calendrier des travaux de la Commission, l'initiative nationale de l'Allemagne consiste simplement à en mettre en relief le caractère urgent pour la dynamique de croissance de l'UE.

Cette insistance est à usage à la fois externe et interne, comme le montre la délicate mise en œuvre de la Directive 'Services'. La tâche est d'une terrible complexité, tant la Directive souffre d'exceptions sectorielles et suscite de cris- 
pations - jusqu'en Allemagne même, où le mouvement syndical (Ver.di en tête) revendique maintenant la création d'un salaire minimum légal (voir REA 79/06) pour parer aux risques de "dumping social ». Le programme 'imposé' comprend aussi la poursuite de la débureaucratisation, notamment au nom de la compétitivité des PME : il faut ramener «l'acquis communautaire » à de plus justes proportions. Cette question a le ferme soutien des fédérations de l'industrie (BDI) et du patronat (BDA) qui voient leurs membres crouler sous la 'paperasse', toutes origines confondues. La chancelière prolonge cet axe en plaidant pour l'adoption d'un "principe de discontinuité » en matière de travaux législatifs. Les projets de directives non votés à la fin de la législature devraient être purement et simplement supprimés; si, après l'élection d'un nouveau PE, le sujet semble encore important, un nouveau texte pourrait toujours être introduit.

\section{Politique énergétique et environnementale : la quadrature du cercle}

La question de la politique énergétique communautaire en relation avec la politique environnementale (et l'avenir du protocole de Kyoto) se révèle particulièrement épineuse pour l'Allemagne : elle la place face à ses propres contradictions. En optant pour l'abandon du nucléaire en 2000 (confirmé par le Traité de grande coalition), l'Allemagne a compromis la stabilité de son Energiemix, au risque de ne plus pouvoir concilier les trois impératifs de toute politique énergétique que sont la sécurité de l'approvisionnement, la compétitivité économique et le respect de l'environnement (voir $R E A 72 / 05)$. Certes, la chancelière affirme que les contraintes environnementales forcent l'innovation. Cela est vrai : la réglementation très stricte sur les émissions adoptée pour l'industrie à partir du milieu des années 1970 avait fait de l'Allemagne un leader en matière d'éco-industries. Mais le contexte des activités a changé, forçant à repenser la relation entre coûts économiques et avantages environnementaux à un moment où se pose en priorité la question de la fiabilité de l'approvisionnement, condition sine qua non pour que les économies génèrent cette croissance indispensable à l'emploi. Un moratoire s'impose pour l'abandon du nucléaire ; certains Länder et électriciens commencent à plaider en ce sens, suivis par le ministre fédéral de l'Economie, Michael Glos (CSU). Quant au ministre fédéral de l'Environnement et de la sécurité des réacteurs nucléaires, Sigmar Gabriel (SPD), il poursuit dans l'UE une politique dédiée aux seuls enjeux climatiques. Pour l'instant, le Contrat de coalition interdit au gouvernement fédéral et à la chancelière de trancher ouvertement, a fortiori dans les enceintes européennes. Au plan intérieur, A. Merkel doit se contenter d'impulser un débat.

En matière sociale, le ministre fédéral des Affaires sociales se contente d'insister sur une meilleure prise en considération de l'intégration des immigrés: l'Allemagne est en train de réviser sa loi sur l'immigration. Les questions sociales ne relèvent pas des compétences communautaires. Et en Allemagne, celles-ci sont partagées. Si le gouvernement porte la responsabilité de l'Etat social, sa mission se résume à déterminer les normes garantissant la cohésion sociale. Pour ce qui est de la politique du travail (rémunérations, durée du travail), les partenaires sociaux sont autonomes et seuls compétents. Dans le cadre de la refondation du modèle économique et social européen, "il importe de donner corps à une politique cohérente reliant étroitement entre eux l'économique, l'emploi et le social - et ce, au même rang de priorité ", rappelait Franz Müntefering (SPD) dans son discours du 9 février tenu à Nuremberg. Dans une déclaration conjointe publiée le 15 novembre 2006, les présidents du DGB (Michael Sommer) et du SPD (Kurt Beck) écrivaient : "La tâche foncière de I'UE est de développer l'économie sociale de marché". Dans sa recommandation à la présidence allemande (05-10-06), le DGB déclarait pour sa part : il faut "générer une croissance durable pour plus d'emplois et de meilleure qualité ". "La politique sociale européenne doit être placée au service de la croissance et de l'emploi ", affirment de leur côté les fédérations BDI et BDA dans un appel commun en date du 8 février. Pour pouvoir redistribuer la richesse, le marché doit être en mesure d'en créer. Mais on est loin d'un libéralisme sauvage : la concurrence doit être régulée, en Europe comme au plan mondial d'ailleurs - c'est là la version allemande, ordo-libérale, d'un 'gouvernement économique' de l'UE. Cette approche partagée outre-Rhin par tous les acteurs, quelle que soit leur obédience (voir REA 78/06), est aussi celle qui fonde le modèle économique et social européen dont la Stratégie de Lisbonne vise à assurer la pérennité.

Dans ce contexte, la 'touche allemande' de la présidence consiste à intégrer plus que de coutume les partenaires sociaux dans le processus de décision européen en matière de réformes structurelles. Si la coopération entre gouvernement et partenaires sociaux est inhérente au « modèle rhénan », elle est sous-
Renforcer l'économie sociale de marché dans l'UE

Développer la participation des partenaires sociaux 
Reconstruire la confiance dans l'Europe

développée à l'échelon communautaire. Le CESE n'a aucun pouvoir décisionnel, et le dialogue entre les partenaires sociaux des Etats membres n'en est qu'à ses balbutiements. Or le projet de Traité constitutionnel apporte sur ce point une avancée fondamentale en renforçant le pouvoir de participation des partenaires sociaux, autrement dit : de la société civile organisée. II va plus loin encore, reconnaissant le rôle d'une société civile dont la responsabilité dépasse celle du simple citoyen.

Plus que tout, la refondation du modèle économique et social européen renvoie à la question du modèle de société désiré par les Européens, donc aux valeurs associées à l'Europe. Or celles-ci ont fini par se brouiller: au fil des générations, les notions 'paix et prospérité' se sont banalisées ; le concept de solidarité a été mis à mal par la dérive des systèmes de protection sociale et une approche trop égalitaire de la cohésion territoriale. L'accélération de l'élargissement de l'Europe, en reléguant au second plan l'approfondissement institutionnel, s'est fait au détriment de la question de la finalité de l'Europe, comme on peut s'en apercevoir dans la méfiance croissante des Européens à l'égard de la politique européenne. L'architecture non équilibrée des institutions communautaires que traduit entre autres la faiblesse du Parlement européen s'est soldée par l'hypertrophie d'une technostructure éloignant encore plus «Bruxelles » des citoyens européens. Pis encore, avoue Peer Steinbrück (SPD), ministre fédéral des Finances, dans son discours du 7 février, les gouvernements nationaux portent une lourde responsabilité dans cette évolution : "quand on cède au populisme en présentant l'UE comme un accélérateur de la mondialisation pour attiser la colère contre le changement qu'imposent le progrès technologique et le vieillissement démographique, on porte préjudice à l'Europe et on contribue dans le même temps à insécuriser encore plus l'opinion ».

CONVAINCRE : VOILA LE CCEUR DE LA MISSION DE L'AlLemAGne. C'est ainsi qu'elle a choisi de comprendre le privilège d'initiative nationale conféré à chaque Etat membre présidant le Conseil. Le succès de sa présidence dépend bien moins d'avancées sur une liste détaillée de dossiers techniques, confie Thomas de Maizière (CDU), Secrétaire d'Etat auprès de la Chancellerie fédérale, que de sa capacité "à sortir l'Europe de la stagnation où elle s'est enfermée » (Die Zeit, 01-02-07). Or cela implique de la part de tous un intense travail de pédagogie nationale pour (re)construire la confiance grâce à un discours honnête et constructif à la fois sur les enjeux européens : "donner une âme à l'Europe », selon la formule de Jacques Delors reprise à son compte par A. Merkel dans son discours du 17 janvier devant le PE. Cela exige aussi de la part de l'Allemagne un comportement exemplaire vis-à-vis de ses partenaires européens comme du monde extérieur. Pendant 6 mois, indépendamment de sa présidence du G8, l'Allemagne représente l'Europe. Et dans ce cadre, la $3^{\text {ème }}$ économie mondiale a une obligation de résultat : réanimer le processus de ratification du Traité constitutionnel - un texte sans lequel l'UE serait condamnée. Elle ne peut miser pour ce faire que sur la force de conviction qu'elle puise dans sa propre histoire depuis qu'elle a scellé son sort à celui de l'Europe - et sur sa capacité à rassembler. Echouer dans cette mission serait pour elle « un manquement à l'Histoire », rappelait A. Merkel devant le Parlement européen.

\section{Indications bibliographiques :}

BouRgeols I. et GABEL M., «Stratégie de Lisbonne - perspectives franco-allemandes », Note de I'Ifri - France, Allemagne, Europe - Perspectives, Paris, février 2007

CAlLIES C., Ruffert M., Verfassung der Europäischen Union. Kommentar der Grundlagenbestimmungen, Munich, 2006

DIE BUNDESREGIERUNG, „Europa gelingt gemeinsam“. Präsidentschaftsprogramm 1. Januar - 30. Juni 2007 (www.eu2007.de)

MAURER A., "Accommoder des attentes contradictoires. Perspectives pour la présidence allemande de l'UE en 2007 ", Analyses et documents, Friedrich-Ebert-Stiftung, fév. 2007 Sturm R., Pehle H., Das neue deutsche Regierungssystem. Die Europäisierung von Institutionen, Entscheidungsprozessen und Politikfeldern in der BRD, Wiesbaden, 2005. 